\title{
Dynamics of Gas Bubbles Encapsulated by a Viscoelastic Fluid Shell Under Acoustic Fields
}

\author{
J. Jiménez-Fernández
}

\begin{abstract}
Summary
The dynamics of a gas-filled microbubble encapsulated by a viscoelastic fluid shell immersed in a Newtonian liquid and subject to an external pressure field is theoretically studied. The problem is formulated by considering a nonlinear Oldroyd type constitutive equation to model the rheological behavior of the fluid shell. Heat and mass transfer across the surface bubble have been neglected but radiation losses due to the compressibility of the surrounding liquid have been taken into account. Bubble collapse under sudden increase of the external pressure as well as nonlinear radial oscillations under ultrasound fields are investigated. The numerical results obtained show that the elasticity of the fluid coating intensifies oscillatory collapse and produces a strong increase of the amplitudes of radial oscillations which may become chaotic even for moderate driving pressure amplitudes. The role played by the elongational viscosity has also been analyzed and its influence on both, bubble collapse and radial oscillations, has been recognized. According to the theoretical predictions provided in the present work, a microbubble coated by a viscoelastic fluid shell is an oscillating system that, under acoustic driving, may experience volume oscillations of large amplitude, being, however, more stable than a free bubble. Thus, it could be expected that such a system may have a suitable behavior as an echogenic agent.
\end{abstract}

\section{Introduction}

The dynamics of free and encapsulated microbubbles is involved in numerous areas and, specially, in the biomedical field, where microbubbles are proven to be very valuable tools as contrast agents for medical ultrasound diagnosis. Indeed, when irradiated by ultrasound fields, these encapsulated bubbles, known as ultrasound contrast agents, enhance the blood-tissue contrast and thereby improve considerably the quality of ultrasonic images $[1,2]$. More recently, they have also been employed for non-invasive therapy and targeted therapeutic drug delivery $[3,4]$. Usually, they consist of a gas core of low solubility which is stabilized against dissolution and coalescence by means of a thin layer of albumin, polymer or lipid material. The mechanical properties of this coating play a dominant role on the overall dynamic behavior of the encapsulated bubble and, therefore, an adequate description of the rheological nature of the shell is a basic aspect of the theoretical analysis.

A great amount of work has been devoted to study the dynamics and sound emission of gas filled encapsulated bubbles immersed in a liquid and irradiated by an acoustic field. Comprehensive reviews have been recently published $[5,6]$. In a first phenomenological approach, as those followed in pioneer works $[7,8]$, a generalized Rayleigh -Plesset equation was formulated by including in the analysis elastic and viscous properties of the encapsulating layer. These coating mechanical properties were introduced by adding to the usual surface tension coefficient, shell elasticity and shell friction terms in the normal stress condition at the gas liquid interface. Other studies have followed a more rigorous analysis based on basic principles of continuous mechanics. A viscoelastic solid layer of finite thickness was considered by Church [9], where the shell was modeled by means of the Kelvin-Voigt rheological equation. The Church model was subsequently simplified in the limit of zero thickness layer by Hoff et al. [10] in order to describe the behavior of bubbles encapsulated by polymeric shells. The dynamics of an encapsulated gas bubble surrounded by a compressible viscoelastic fluid was investigated by Khismatullin and Nadim [11]. In this work, the shell was also modeled by the KelvinVoigt rheological equation and it was shown that the elastic and viscous properties of the shell are dominant over those of the host fluid.

Some approaches including non-linear constitutive equations to describe the rheological behavior of the shell have also been considered. The Mooney-Rivlin constitutive law along with the Skalak model were investigated by Tsiglifis and Pelekasis [12] in order to describe strain-softening as well as strain-hardening elastic behav- 
iors respectively. The numerical results provided in that work, predict striking differences between both behaviors. The Mooney-Rivlin model has been also used to predict the behavior observed in acoustic experiments on lipidshelled microbubbles [13]. The constitutive equation of an isotropic hyperelastic neo-Hookean material has been considered by Allen and Rashid [14] to analyze the mechanical problem. More recently, the acoustic backscatter of bubbles encapsulated by a solid coating modeled by this rheological equation has been investigated by Jiménez-Fernández [15] in order to evaluate harmonic resonance frequencies in nonlinear conditions. Alternative approaches where the coating is described as a surfactant layer, have been proposed $[16,17,18,19]$. The assumption of an homogeneous and isotropic layer has been revised by Chatterjee and Sarkar [17], and Sarkar et al. [18]. They have proposed a different approach based on interfacial models with intrinsic surface rheology. In the same line, in the model of Stride [19], it is assumed that the bubble is encapsulated by a homogeneous molecular monolayer with surface tension and interfacial viscosity depending on the surface molecular concentration.

As pointed out by Doinikov and Bouakaz [5] the interfacial models described above, as well as, those based on the Kelvin-Voigt equation, lead to similar results for oscillations of infinitesimal amplitude (linear domain). Thus, the constant shell parameters have been usually estimated by fitting the theoretical results provided by linear analysis, with experimental data corresponding to frequencydependent attenuation or radius time curves.

Nevertheless, these theoretical approaches have been challenged by several experimental observations, some of which, are even detected for acoustic pressures as low as $10 \mathrm{kPa}$. Among these, it should be firstly quoted the effect known as "compression-only" behavior, where the bubble experiences a noticeable compression phase but hardly expands [20]. Another effect observed is the "thresholding" behavior, that is, the existence of an acoustic pressure threshold for the onset of bubble oscillations [21, 22, 23]. Finally, other not much explained effect is the experimentally observed dependence of shell elasticity and shell viscosity on the initial bubble radius $[16,24,25]$. These experimental results have motivated a revision of previous theoretical analysis and more complex rheological models have been proposed. Some of the experimental observations quoted above have been appropriately described by the model proposed by Marmottant et al. [26], a heuristic model based on the behavior of phospholipid monolayer coating with surface tension depending on bubble area which takes into account shell buckling and rupture. By assuming that a lipid coating may be a material which exhibit both, shear thinning and strain-softening behavior, Doinikov et al. [25] have developed a nonlinear model which predict, in good agreement with experimental results, compression only behavior. Two nonlinear interfacial models have been formulated by Paul et al. [27] which under some conditions also predict compression only behavior. Sijl et al. [28] have shown with the aid of the
Marmottant model through a weakly nonlinear analysis that compression only behavior may result from a sudden change of shell elasticity with bubble radius. The Marmottant model has been recently extended by considering the nonlinear Cross-law for the shell viscous term [29] in order to introduce shear-thinning behavior.

For liquid coating the problem has been less explored. For a class of encapsulated microbubbles used in therapeutic applications, a Newtonian fluid layer was considered by Allen et al. [30]. For lipid coating, the equation of a linear Maxwell fluid has been considered by Doinikov and Dayton [31] in an attempt to explain some experimental results which show a considerable increase of the resonance frequency of encapsulated bubbles compared with free bubbles.

In this work, the dynamics of gas-filled bubbles encapsulated by a viscoelastic fluid shell immersed in an infinite Newtonian liquid and subject to an external pressure field is theoretically investigated. The problem is formulated by considering for the fuid shell, a nonlinear Oldroyd type constitutive equation with an interpolated time derivative $[32,33]$. Heat and mass transfer across the encapsulating layer has been neglected but radiation losses due to the compressibility of the surrounding liquid have been taken into account. Bubble collapse under sudden increase of the external ambient pressure as well as nonlinear radial oscillations under monochromatic acoustic fields are analyzed. The objective of the work is to determine the quantitative influence of the elastic as well as viscous properties of the fluid shell on bubble collapse and bubble radial oscillations.

The numerical results obtained show that fluid elasticity enhances considerably the amplitude of the radial oscillations. In fact, chaotic behavior is predicted for moderate pressure amplitudes although critical values for bifurcation and chaos are larger than those corresponding to a free bubble. On the other hand, the role played by the elongational viscosity in both bubble collapse and bubble oscillations has been investigated.

Besides eventual applications in other areas, the results obtained in the present analysis may be of particular interest in biomedical applications. Indeed, according to the theoretical predictions provided in this work, it may be concluded that a bubble encapsulated by a viscoelastic fluid shell could have an excellent behavior as an echogenic agent.

The full paper is organized as follows: In section 2 the equations governing the radial motion are formulated according to the Oldroyd rheological model. Results are presented in section 3. Section 3.1 is devoted to investigate the bubble collapse under a sudden increase of the ambient pressure. In section 3.2 bubble oscillations driven by a monochromatic acoustic wave are analyzed. Dynamic behavior and transition to chaos are described in section 3.3. In section 3.4 the dependence of the maximal amplitude expansion on the governing parameters is determined. Finally in section 4 the main conclusion of the work are summarized. 


\section{The mathematical model}

\subsection{The equations for radial motions}

Let us consider a gas bubble encapsulated by a viscoelastic shell and immersed in a Newtonian liquid of density $\rho_{l}$ and viscosity $\eta_{l}$. In a spherical coordinate system $\{r, \theta, \phi\}$ with its origin at the center of the spherical bubble, the instantaneous inner and outer radii are denoted by $r_{1}$ and $r_{2}$ respectively. It is assumed that the volumetric bubble motion is purely radial so that spherical symmetry is held at any time. It is also considered that the encapsulating layer is incompressible and therefore,

$$
r_{2}^{3}-r_{1}^{3}=R_{2}^{3}-R_{1}^{3},
$$

where $R_{1}$ and $R_{2}$ are the initial inner and outer radii respectively.

Following a similar analysis to the one performed in [15], the equations of motion in the viscoelastic shell are integrated between $r_{1}$ and $r_{2}$ to obtain

$\rho_{s}\left[\varphi\left(r_{1}\right)-\varphi\left(r_{2}\right)\right]=T_{r r}\left(r_{2}\right)-T_{r r}\left(r_{1}\right)+\Gamma\left(r_{1}, t\right)$,

where $\rho_{s}$ is the density of the shell, $T_{r r}$ is the $r r$-component of the stress tensor in the shell $\mathbf{T}$, and $\varphi(r)$ is the potential,

$$
\varphi(r)=\frac{1}{r} \frac{\partial}{\partial t}\left(\dot{r}_{1} r_{1}^{2}\right)-\frac{\left(\dot{r}_{1} r_{1}^{2}\right)^{2}}{2 r^{4}}
$$

where the dot denotes differentiation with time and $\Gamma\left(r_{1}\right)$ is the integral

$$
\Gamma\left(r_{1}, t\right)=2 \int_{r_{1}}^{r_{2}} \frac{\tau_{r r}-\tau_{\theta \theta}}{r} \mathrm{~d} r .
$$

$\tau_{r r}, \tau_{\theta \theta}$ are, respectively, the $r r$-component and the $\theta \theta$ component of the extra stress tensor $\boldsymbol{\tau}=\mathbf{T}+p \mathbf{I}$ in the shell, $p$ is the isotropic pressure and $\mathbf{I}$ is the identity tensor.

$T_{r r}\left(r_{1}\right)$ and $T_{r r}\left(r_{2}\right)$ are determined from normal stress balances at the inner and outer interface respectively. Thus, at $r=r_{1}$,

$$
T_{r r}\left(r_{1}\right)=-p_{g}\left(r_{1}\right)+\frac{2 \sigma_{1}}{r_{1}},
$$

where $p_{g}=p_{g 0}\left(r_{1} / R_{1}\right)^{3 \gamma}$ is the gas pressure inside the bubble, $p_{g 0}$ the gas pressure at the initial state, $\gamma$ the polytropic index and $\sigma_{1}$ the surface tension coefficient at the gas-shell surface. Likewise, a stress balance at the external interphase $r=r_{2}$ gives

$$
T_{r r}\left(r_{2}\right)=T_{r r}^{l}\left(r_{2}\right)-\frac{2 \sigma_{2}}{r_{2}},
$$

where $\sigma_{2}$ is the surface tension coefficient at the shellliquid surface and $T_{r r}^{l}$, the $r r$-component of the stress tensor in the liquid phase $\mathbf{T}^{\mathbf{l}} \cdot T_{r r}^{l}\left(\boldsymbol{r}_{2}\right)$ is determined by integration of the motion equations in the external liquid phase. In the present analysis, heat transfer across the surface layer has been neglected but compressibility effects in the liquid phase have been taken into account. Indeed, linear analysis for encapsulated microbubbles have shown that thermal and acoustic damping are negligible with respect to viscous damping $[9,11]$. Nevertheless, as it has been noted by Doinikov-Boukaz [5], no available analysis provides whether these conclusions on oscillations of infinitesimal amplitude hold for non-linear oscillations. Moreover, apart from the relative importance of thermal and acoustic damping on the total damping constant, it should be emphasized, that for micrometer bubble radius size and megahertz frequencies, the bubble shell wall velocity can reach values high enough to invalid the assumption of an incompressible liquid host. In other words, the effects of acoustic radiation may be of substantial importance for large amplitude bubble oscillations. Consequently, in the present work a boundary layer analysis analogous to those performed by Prosperetti and Lezzi [34] for a free bubble and by Khismatullin and Nadim [11] for an encapsulated bubble, has been carried out in order to include compressibility effects in the liquid phase. Accordingly, the following expression for $T_{r r}^{l}\left(r_{2}\right)$ has been obtained,

$T_{r r}^{l}\left(r_{2}\right)=-4 \eta_{l} \frac{\dot{r}_{2}}{r_{2}}-\rho_{l} \varphi\left(r_{2}\right)-p_{\infty}+\frac{\rho_{l}}{c_{s}} \frac{\mathrm{d}^{2}}{\mathrm{~d} t^{2}}\left(r_{2}^{2} \dot{r}_{2}\right)$,

where $p_{\infty}$ is the pressure far away from the bubble (a constant value or a monochromatic wave) and $c_{s}$ the sound velocity in the liquid phase. Finally, by substitution of expressions (3) to (7) into expression (2) the following governing equation for the bubble radial motion is obtained,

$$
\begin{aligned}
\rho_{s} r_{1} \ddot{r}_{1} & {\left[1+\chi_{1}-\frac{\rho_{l}}{\rho_{s} c_{s}} \dot{r}_{1} \chi_{2}\right] } \\
+\rho_{S} \dot{r}_{1}^{2} & {\left[\frac{3}{2}+\chi_{1}\left(2-\frac{r_{1}^{3}}{2 r_{2}^{3}}\right)-\frac{\rho_{l}}{\rho_{s} c_{S}} \frac{1}{2} \frac{d}{d t} r_{1} \chi_{2}\right] } \\
= & \left(p-p_{\infty}\right)+\frac{\rho_{l}}{\rho_{S} c_{s}} \frac{\mathrm{d}}{\mathrm{d} t} \frac{r_{1}}{1+\chi_{1}}\left(p-p_{\infty}\right),
\end{aligned}
$$

where

$$
\chi_{1}\left(r_{1}\right)=\frac{\rho_{l}-\rho_{s}}{\rho_{s}} \frac{r_{1}}{r_{2}}, \quad \chi_{2}\left(r_{1}\right)=\frac{1+\chi_{1} r_{1}^{3} / r_{2}^{3}}{1+\chi_{1}},
$$

and

$$
p=p_{g}-\left(\frac{2 \sigma_{1}}{r_{1}}+\frac{2 \sigma_{2}}{r_{2}}\right)-4 \eta_{l} \frac{\dot{r}_{2}}{r_{2}}+\Gamma .
$$

\subsection{The constitutive equation for the shell}

The function $\Gamma\left(r_{1}, t\right)$, as defined in (4), should be determined from a constitutive equation for the extra stress tensor $\boldsymbol{\tau}$. In this analysis, a nonlinear Oldroyd type equation [33] will be considered. This rheological model is defined by the equation

$$
\begin{aligned}
& \tau+\lambda_{1}\left(\frac{\check{D} \tau}{\check{D} t}-a\left(\tau^{s} \cdot e+e \cdot \tau\right)\right) \\
& =2 \eta e+2 \eta \lambda_{2}\left(\frac{\check{D} e}{\check{D} t}-2 a e \cdot e\right),
\end{aligned}
$$


where $\eta$ is the shell viscosity, $e=\frac{1}{2}\left(\nabla v+(\nabla v)^{T}\right)$ the rate of strain tensor and $\nabla \boldsymbol{v}$ the velocity gradient tensor. $\lambda_{1}$ is the stress relaxation time and $\lambda_{2}$ the retardation time. $\check{D} / \check{D} t$ is the Jaumann derivative given for an arbitrary second order tensor $\beta$ by: $\breve{D} \beta / \check{D} t=\partial \beta / \partial t+\boldsymbol{v} \nabla \boldsymbol{\beta}+\boldsymbol{\beta} \boldsymbol{w}-\boldsymbol{w} \boldsymbol{\beta}$ where $\boldsymbol{w}$ is the vorticity tensor that for this irrotational flow vanishes. The term in large brackets in expression (11), known as the Gordon-Schowalter derivative, is an invariant derivative for any value of $a$ in the interval: $-1 \leq a \leq 1$. [35]. Depending on the values assigned to the parameter $a$, different rheological responses are predicted. For $a=1$ expression (11) provides the Oldroyd$B$ model which reduces to the upper convected Maxwell model (UCM model) if $\lambda_{2}=0$. The Oldroyd-B model ( $a=1$ ), as well as the Oldroyd-A model $(a=-1)$ predict a constant shear viscosity and an unbounded elongational viscosity, however, for intermediate values of $a$ the above model exhibits both extensional viscosity depending on the extensional rate and shear thinning viscosity.

\subsection{The equations of motion for the Oldroyd model}

It is convenient decompose the extra stress tensor in the shell $\tau$ in the form $\tau=\tau^{S}+\tau^{N}$, where $\tau^{N}=2 \eta_{N} \mathrm{e}$, is a Newtonian contribution with $\eta_{N}=\eta \lambda_{2} / \lambda_{1}$ and $\tau^{s}$ satisfies the equation

$$
\tau^{s}+\lambda_{1}\left(\frac{\check{D} \tau^{s}}{\check{D} t}-a\left(\tau^{s} \cdot e+e \cdot \tau^{s}\right)\right)=2 \eta_{s} e,
$$

where $\eta_{s}=\eta-\eta_{N}=\eta\left(1-\lambda_{2} / \lambda_{1}\right)$. For polymeric solutions, $\eta_{N}$ may be regarded like the viscosity of a Newtonian solvent and $\eta_{s}$ the polymer viscosity, so that $\eta=$ $\eta_{s}+\eta_{N}$ is the total solution viscosity. According to the above decomposition, equation (4) may be written in the form

$$
\Gamma\left(r_{1}, t\right)=-4 \eta_{N} \frac{\dot{r}_{1} V}{\dot{r}_{2}^{3} r_{1}}+S\left(r_{1}, t\right),
$$

where $V=R_{2}^{3}-R_{1}^{3}$ is the constant volume of the shell and $S\left(r_{1}, t\right)$ is defined as

$$
S\left(r_{1}, t\right)=2 \int_{r_{1}}^{r_{2}} \frac{\tau_{r r}^{s}-\tau_{\theta \theta}^{s}}{r} \mathrm{~d} r .
$$

Consider the Lagrangian coordinate transformation,

$$
3 h=r^{3}-r_{1}^{3} .
$$

After this transformation, expression (14) is written

$$
\begin{aligned}
S\left(r_{1}, t\right) & =2 \int_{r_{1}}^{r_{2}} \frac{\tau_{r r}^{s}-\tau_{\theta \theta}^{s}}{r} \mathrm{~d} r \\
& =2 \int_{0}^{V / 3} \frac{\tau_{r r}^{s}-\tau_{\theta \theta}^{s}}{3 h+r_{1}^{3}} \mathrm{~d} h,
\end{aligned}
$$

and $\tau_{r r}$ and $\tau_{\theta \theta}$ are obtained as solution of the equations

$$
\begin{aligned}
\tau_{r r}^{s}+\lambda_{1}\left(\frac{\mathrm{d} \tau_{r r}^{s}}{\mathrm{~d} t}-a \varepsilon(t) \tau_{r r}^{s}\right) & =2 \eta_{s} \varepsilon(t), \\
\tau_{\theta \theta}^{s}+\lambda_{1}\left(\frac{\mathrm{d} \tau_{\theta \theta}^{s}}{\mathrm{~d} t}+a \varepsilon(t) \tau_{\theta \theta}^{s}\right) & =-\eta_{s} \varepsilon(t),
\end{aligned}
$$

where

$$
\varepsilon(t)=-\frac{2 r_{1}^{2} \dot{r}_{1}}{3 h+r_{1}^{3}} .
$$

Integration of the above equations from $t^{\prime}=0$ to $t^{\prime}=t$, leads to

$$
\tau_{r r}^{s}=-\frac{4 \eta_{p}}{\lambda_{1}} \int_{0}^{t} \mathrm{e}^{\frac{t^{-}-t}{\lambda_{1}}} r_{1}^{2}\left(t^{\prime}\right) \dot{r}_{1}\left(t^{\prime}\right) \frac{\left(3 h+r_{1}^{3}\right)^{\frac{4 a}{3}-1}}{\left(3 h+r_{1}^{3}\right)^{\frac{4 a}{3}}} \mathrm{~d} t^{\prime}
$$

and

$$
\tau_{\theta \theta}^{s}=\frac{2 \eta_{p}}{\lambda} \int_{0}^{t} \mathrm{e}^{\frac{t^{\prime}-t}{\lambda_{1}}} r_{1}^{2}\left(t^{\prime}\right) \dot{r}_{1}\left(t^{\prime}\right) \frac{\left(3 h+r_{1}^{3}\right)^{\frac{2 a}{3}}}{\left(3 h+r_{1}^{3}\right)^{\frac{2 a}{3}+1}} \mathrm{~d} t^{\prime} .
$$

Replacing above expressions in equation (16) gives

$$
S\left(r_{1}, t\right)=\frac{2 \eta_{p}}{\lambda_{1}} \int_{0}^{t} \mathrm{e}^{\frac{t^{t}-t}{\lambda_{1}}} r_{1}^{2}\left(t^{\prime}\right) \dot{r}_{1}\left(t^{\prime}\right)\left[\Psi_{1}-\Psi_{2}\right] \mathrm{d} t^{\prime},
$$

where

$$
\begin{aligned}
\Psi_{1} & =\int_{0}^{V / 3} \frac{\tau_{r r}^{s}}{3 h+r_{1}^{3}} \mathrm{~d} h \\
& =\frac{1}{4 a\left[r_{1}^{3}(t)-r_{1}^{3}\left(t^{\prime}\right)\right]}\left[\left(\frac{r_{2}^{3}\left(t^{\prime}\right)}{r_{2}^{3}(t)}\right)^{4 a}-\left(\frac{r_{1}^{3}\left(t^{\prime}\right)}{r_{1}^{3}(t)}\right)^{4 a}\right]
\end{aligned}
$$

and

$$
\begin{aligned}
\Psi_{2} & =\int_{0}^{V / 3} \frac{\tau_{\theta \theta}^{s}}{3 h+r_{1}^{3}} \mathrm{~d} h \\
& =-\frac{1}{2 a\left[r_{1}^{3}(t)-r_{1}^{3}\left(t^{\prime}\right)\right]}\left[\left(\frac{r_{2}^{3}(t)}{r_{2}^{3}\left(t^{\prime}\right)}\right)^{2 a}-\left(\frac{r_{1}^{3}(t)}{r_{1}^{3}\left(t^{\prime}\right)}\right)^{2 a}\right] .
\end{aligned}
$$

For any value of the parameter $a$, expressions (13), (22)(24) introduced in equations (8)-(10) lead to an integrodifferential equation for $r_{1}$ whose resolution presents difficult problems for arbitrary values of the governing parameters. However, as it has been previously shown by Shulman and Levitskii [36] and Jiménez-Fernández and Crespo [37], for $a=1 / 2$ or $a=1$ (Oldroyd-B model) this integrodifferential equation may be reduced to an ordinary differential system. Indeed, for $a=1 / 2$, equation (22) may be written in the form: $S(t)=S_{1}(t)+S_{2}(t)$, where

$$
S_{1}(t)=-\frac{4 \eta_{p}}{\lambda_{1} r_{1}^{2}(t)} \int_{0}^{t} \mathrm{e}^{\frac{t^{-}-t}{\lambda_{1}}} r_{1}\left(t^{\prime}\right) \dot{r}_{1}\left(t^{\prime}\right) \mathrm{d} t^{\prime}
$$

and

$$
S_{2}(t)=\frac{4 \eta_{p}}{\lambda_{1} r_{2}^{2}(t)} \int_{0}^{t} \mathrm{e}^{\frac{t^{\prime}-t}{\lambda_{1}}} \frac{r_{1}^{2}\left(t^{\prime}\right) \dot{r}_{1}\left(t^{\prime}\right)}{r_{2}\left(t^{\prime}\right)} \mathrm{d} t^{\prime}
$$


Derivation with respect to time and ulterior application of the Leibniz theorem reduce the above expressions to the ordinary differential system

$$
\begin{aligned}
& \dot{S}_{1}=-\left(\frac{1}{\lambda_{1}}+2 \frac{r_{1}}{r_{1}}\right) S_{1}-4 \frac{\eta_{s}}{\lambda_{1}} \frac{\dot{r}_{1}}{r_{1}}, \\
& \dot{S}_{2}=-\left(\frac{1}{\lambda_{1}}+2 \frac{r_{2}}{r_{2}}\right) S_{2}+4 \frac{\eta_{s}}{\lambda_{1}} \frac{\dot{r_{2}}}{r_{2}} .
\end{aligned}
$$

Likewise for $a=1$ (Oldroyd-B) it is obtained $S(t)=$ $S_{11}(t)+S_{12}(t)+S_{21}(t)+S_{22}(t)$, where

$$
\begin{aligned}
& \dot{S}_{11}=-\left(\frac{1}{\lambda_{1}}-\frac{\dot{r_{1}}}{r_{1}}\right) S_{11}-2 \frac{\eta_{s}}{\lambda_{1}} \frac{\dot{r}_{1}}{r_{1}}, \\
& \dot{S}_{12}=-\left(\frac{1}{\lambda_{1}}+2 \frac{\dot{r_{1}}}{r_{1}}\right) S_{12}-2 \frac{\eta_{s}}{\lambda_{1}} \frac{\dot{r_{1}}}{r_{1}}, \\
& \dot{S}_{21}=-\left(\frac{1}{\lambda_{1}}-\frac{\dot{r_{2}}}{r_{2}}\right) S_{21}+2 \frac{\eta_{s}}{\lambda_{1}} \frac{\dot{r_{2}}}{r_{2}}, \\
& \dot{S}_{22}=-\left(\frac{1}{\lambda_{1}}+2 \frac{\dot{r_{2}}}{r_{2}}\right) S_{22}+2 \frac{\eta_{s}}{\lambda_{1}} \frac{\dot{r_{2}}}{r_{2}} .
\end{aligned}
$$

The above reduction to a differential system may be also accomplished for $a=-1$ (Oldroyd-A). Indeed, following a similar procedure it has been obtained

$$
\begin{aligned}
& \dot{S}_{11}=-\left(\frac{1}{\lambda_{1}}+4 \frac{\dot{r_{1}}}{r_{1}}\right) S_{11}-2 \frac{\eta_{s}}{\lambda_{1}} \frac{\dot{r_{1}}}{r_{1}} . \\
& \dot{S}_{12}=-\left(\frac{1}{\lambda_{1}}+\frac{\dot{r_{1}}}{r_{1}}\right) S_{12}-2 \frac{\eta_{s}}{\lambda_{1}} \frac{\dot{r_{1}}}{r_{1}}, \\
& \dot{S}_{21}=-\left(\frac{1}{\lambda_{1}}+4 \frac{\dot{r_{2}}}{r_{2}}\right) S_{21}+2 \frac{\eta_{s}}{\lambda_{1}} \frac{\dot{r_{2}}}{r_{2}} . \\
& \dot{S}_{22}=-\left(\frac{1}{\lambda_{1}}+\frac{\dot{r_{2}}}{r_{2}}\right) S_{22}+2 \frac{\eta_{s}}{\lambda_{1}} \frac{\dot{r_{2}}}{r_{2}} .
\end{aligned}
$$

The system (8)-(10) with $\Gamma\left(r_{1}, t\right)$ defined in (13) provides the governing equations for the radial bubble motion. The function $S\left(r_{1}, t\right)$ is determined for $a=1 / 2$ by the differential system (27)-(28), for $a=1$ by the differential system (29) $-(32)$ and for $a=-1$ by the differential system (33)-(36), respectively. These systems are written in dimensionless form by introducing the scales: length: $\boldsymbol{R}_{1}$, time: $R_{1} \sqrt{p_{S} / p_{c}}$, and pressure $p_{c}:$ i) a constant value far away from the bubble $p_{\infty}$ for the collapse problem or ii) the ambient pressure $p_{0}$ when the external pressure is a monochromatic wave. Accordingly, the following non dimensional equations are obtained,

$$
\begin{aligned}
& f_{1}\left(x_{1}\right) x_{1} \ddot{x}_{1}+f_{2}\left(x_{1}\right) \dot{x}_{1}^{2}= \\
& p^{*}-p_{\infty}^{*}+\kappa M \frac{\mathrm{d}}{\mathrm{d} t^{*}} \frac{x_{1}\left(p^{*}-p_{\infty}^{*}\right)}{1+f\left(x_{1}\right)} .
\end{aligned}
$$

where $f_{1}\left(x_{1}\right)=1+f\left(x_{1}\right)-\lambda M F\left(x_{1}\right) \dot{x}_{1}$,

$$
\begin{aligned}
f_{2}\left(x_{1}\right)= & \frac{3}{2}+f\left(x_{1}\right)\left(2-\frac{x_{1}^{3}}{2 x_{2}^{3}}\right)-\frac{1}{2} \kappa M \frac{\mathrm{d}}{\mathrm{d} t^{*}} x_{1} F\left(x_{1}\right), \\
f\left(x_{1}\right)= & (\kappa-1) \frac{x_{1}}{x_{2}}, \quad F\left(x_{1}\right)=\frac{1+(\kappa-1)\left(\frac{x_{1}}{x_{2}}\right)^{4}}{1+(\kappa-1) \frac{x_{1}}{x_{2}}}, \\
p^{*}\left(x_{1}\right)= & q\left(\frac{1}{x_{1}}\right)^{3 \gamma}-2\left(\frac{W e_{1}}{x_{1}}+\frac{W e_{2}}{x_{2}}\right) \\
& -\frac{4}{R e_{l}} \frac{\dot{x}_{2}}{x_{2}}-\frac{4 \varepsilon}{R e_{s}} \frac{\dot{x}_{1}\left(d^{3}-1\right)}{x_{2}^{3} x_{1}}+S^{*}
\end{aligned}
$$

and $S^{*}$ is given for the following systems depending on the values of the parameter $a$,

for $a=1 / 2$ :

$$
\begin{aligned}
& S^{*}=S_{1}^{*}+S_{2}^{*} \\
& \dot{S}_{1}^{*}+\left(\frac{1}{D e}+2 \frac{\dot{x_{1}}}{x_{1}}\right) S_{1}^{*}=-4 \frac{(1-\varepsilon)}{D e e_{s}} \frac{x_{1}}{x_{1}} \\
& \dot{S}_{2}^{*}+\left(\frac{1}{D e}+2 \frac{\dot{x_{2}}}{x_{2}}\right) S_{2}^{*}=4 \frac{(1-\varepsilon)}{D e R e_{s}} \frac{\dot{x}_{2}}{x_{2}},
\end{aligned}
$$

for $a=1$ :

$$
\begin{aligned}
& S^{*}=S_{11}^{*}+S_{12}^{*}+S_{21}^{*}+S_{22}^{*}, \\
& \dot{S}_{11}^{*}+\left(\frac{1}{D e}+4 \frac{\dot{x_{1}}}{x_{1}}\right) S_{11}^{*}=-2 \frac{(1-\varepsilon)}{D e e_{s}} \frac{\dot{x_{1}}}{x_{1}}, \\
& \dot{S}_{12}^{*}+\left(\frac{1}{D e}+\frac{\dot{x_{1}}}{x_{1}}\right) S_{12}^{*}=-2 \frac{(1-\varepsilon)}{D e e_{s}} \frac{\dot{x_{1}}}{x_{1}}, \\
& \dot{S}_{21}^{*}+\left(\frac{1}{D e}+4 \frac{\dot{x_{2}}}{x_{2}}\right) S_{21}^{*}=2 \frac{(1-\varepsilon)}{D e e_{s}} \frac{\dot{x_{2}}}{x_{2}}, \\
& \dot{S}_{22}^{*}+\left(\frac{1}{D e}+\frac{\dot{x_{2}}}{x_{2}}\right) S_{22}^{*}=2 \frac{(1-\varepsilon)}{D e R e_{s}} \frac{\dot{x_{2}}}{x_{2}},
\end{aligned}
$$

and for $a=-1$ :

$$
\begin{gathered}
\dot{S}_{11}^{*}+\left(\frac{1}{D e}-\frac{x_{1}}{x_{1}}\right) S_{11}^{*}=-2 \frac{(1-\varepsilon)}{D e e_{s}} \frac{\dot{x}_{1}}{x_{1}}, \\
\dot{S}_{12}^{*}+\left(\frac{1}{D e}+2 \frac{\dot{x_{1}}}{x_{1}}\right) S_{12}^{*}=-2 \frac{(1-\varepsilon)}{D e \operatorname{Re} s} \frac{\dot{x_{1}}}{x_{1}} \\
\dot{S}_{21}^{*}+\left(\frac{1}{D e}-\frac{\dot{x_{2}}}{x_{2}}\right) S_{21}^{*}=2 \frac{(1-\varepsilon)}{D e e_{s}} \frac{\dot{x_{2}}}{x_{2}}, \\
\dot{S}_{22}^{*}+\left(\frac{1}{D e}+2 \frac{\dot{x_{2}}}{x_{2}}\right) S_{22}^{*}=2 \frac{(1-\varepsilon)}{D e \operatorname{Re}_{s}} \frac{\dot{x_{2}}}{x_{2}} .
\end{gathered}
$$

Stars, which will be suppressed hereafter, denote dimensionless quantities, $x_{1}=r_{1} / R_{1}, x_{2}=r_{2} / R_{1}, \kappa=\frac{p_{l}}{\rho_{s}}$, 
$d=R_{2} / R_{1}, M=\sqrt{p_{c}} / c_{s} \sqrt{\rho_{s}}$ is the Mach number, $q=p_{g 0} / p_{c}, W e_{1}=2 \sigma_{1} / R_{1} p_{c}, W e_{2}=2 \sigma_{2} / R_{1} p_{c}$ are the Weber numbers for the internal and external interfaces respectively, $R e_{l}=\rho_{l} R_{1} \sqrt{p_{c}} /\left(\eta_{l} \sqrt{\rho_{s}}\right)$ is the Reynolds number in the liquid phase, $R e_{s}=R_{1} \sqrt{p_{c} \rho_{s}} / \eta$ the Reynolds number in the shell, $D e=\lambda_{1} / t_{c}$ the Deborah number and $\varepsilon=\lambda_{2} / \lambda_{1}$.

\section{Results and discussion}

\subsection{Bubble collapse}

Consider that the bubble, at rest at the external pressure $p_{0}$ for instant times prior to zero, is driven out of equilibrium when the external pressure is suddenly increased to a constant value $p_{\infty}$, so that the bubble evolves to a new equilibrium state. In order to analyze this dynamic process, the system (37)-(40) along with the appropriated system associated to $S\left(x_{1}, t\right)$ for any value of $a$, will be numerically solved along with the initial conditions: $x_{1}(0)=1$, $\dot{x}(0)=0, S\left(x_{1}, 0\right)=0$. In the following, it will be considered that the host fluid is water and, therefore, results will be obtained by fixing the values: $\rho_{l}=1000 \mathrm{Kg} / \mathrm{m}^{3}$, $\eta_{l}=0.001 \mathrm{Pas}, c_{s}=1450 \mathrm{~m} / \mathrm{s}$ and the ambient pressure $p_{0}=1.013 \times 10^{5} \mathrm{~Pa}$. In accordance with the experimental data available for ultrasound contrast agents provided in previous works on encapsulated bubble dynamics $[9,11]$, it will also be considered as fixed values in ulterior calculations, the equilibrium inner radius $R_{1}=2 \mu \mathrm{m}$, the polytropic index $\gamma=1.4$, the shell density $\rho_{s}=1100 \mathrm{Kg} / \mathrm{m}^{3}$, and the surface tension coefficients: $\sigma_{1}=0.04 \mathrm{~N} / \mathrm{m}, \sigma_{2}=0.005 \mathrm{~N} / \mathrm{m}$. Regarding the shell viscosity $\eta$ and the relaxation time $\lambda_{1}$, the estimated values provided by Doinikov and Dayton [31] for lipid-shelled microbubbles will be here adopted. As it is described in this work, the relaxation time $\lambda_{1}$ may be estimated by the quotient between the shell viscosity $\eta$ and the shell elasticity modulus $G$ corresponding to the Kelvin-Voigt model. In turn, the parameters $G$ and $\eta$ may be related, in the linear domain, with the experimental data available for the dilatational viscosity and the elastic compression modulus involved in interfacial models. Accordingly, shell viscosity values in the range: $0.5-1.75 \mathrm{Pas}$, and relaxation time values in the range: $0.01-1$ us will be, respectively, considered. The retardation time $\lambda_{2}$ appears in the analysis through the quotient $\lambda_{2} / \lambda_{1}$. This one must be greater than $1 / 9$ to assure that the shear stress increases monotonically with increasing shear rate for steady shear flows [32]. Thus, a minimum value $\varepsilon=\lambda_{2} / \lambda_{1}=1 / 9$ will be considered for all calculations.

In this section (Figures 1-5) results will be obtained for a pressure ratio $q=p_{0} / p_{\infty}=0.01$, a shell viscosity $\eta=$ $0.5 \mathrm{Pas}$ and a layer thickness $R_{2}-R_{1}=2 \mathrm{~nm}$, except for Figure $3 \mathrm{~b}$, where $\eta=1.75 \mathrm{~Pa}$ and $R_{2}-R_{1}=10 \mathrm{~nm}$.

In Figure 1 results are shown for the collapse of a freebubble, a Newtonian shell $(D e=0)$ and a viscoelastic fluid shell with $D e=1$ and $a=1 / 2$. To illustrate the contrast with an elastic solid layer, results for a Kelvin-

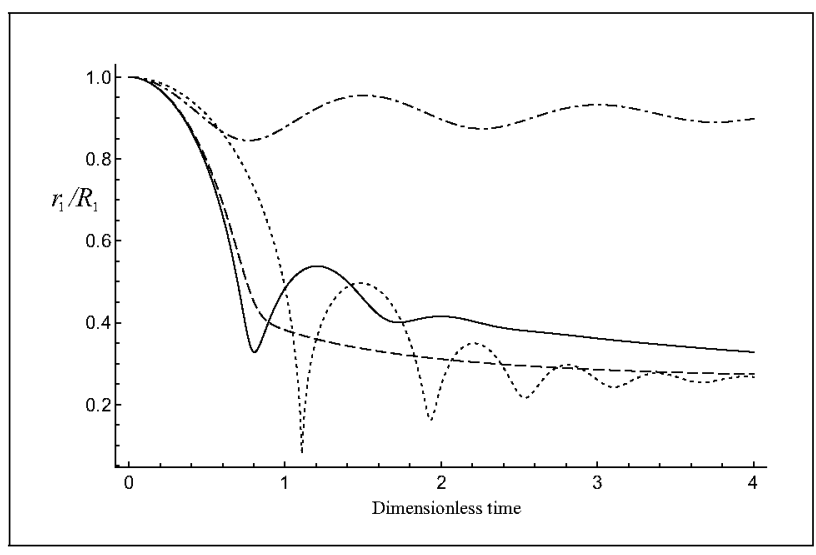

Figure 1. Bubble collapse: normalized inner radius $r_{1} / R_{1}$ vs dimensionless time. Solid line: viscoelastic fluid shell with $D e=1$, dashed line: Newtonian fluid shell ( $D e=0)$, dotted line: freebubble, dash-dotted line: elastic Kelvin-Voigt shell with $G=$ $50 \mathrm{MPa}$.

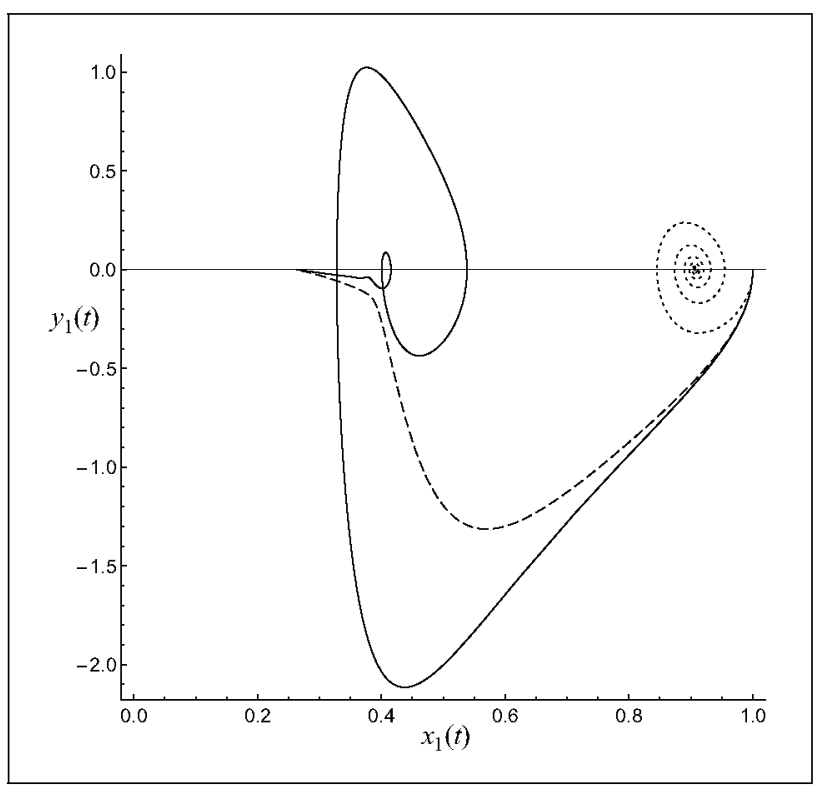

Figure 2. Bubble collapse: trayectories in the phase space. Solid line: viscoelastic fluid shell with $D e=1$, dashed line: Newtonian fluid shell $(D e=0)$, dotted line: elastic Kelvin-Voigt shell with $G=50 \mathrm{MPa}$.

Voigt shell according to the formulation outlined in a previous work [15], with a shear modulus $G=50 \mathrm{MPa}$ have also been included in the same figure. As it may be observed at first sight, for the parameter values considered, an oscillatory behavior similar to that observed for free bubbles, is predicted for a viscoelastic shell whereas the collapse is monotonous for a Newtonian shell. Note, however, the damping influence of the shell which produces a clear reduction of the amplitude of radial oscillations. This time evolution to new equilibrium states is shown in Figure 2 where trayectories in the phase space have been plotted. In the elastic case the bubble follows a damped oscillatory trayectory towards a equilibrium state close to the initial one. By contrast, for a viscoelastic fluid shell the integrated normal stress difference $S$ tends to zero for 
$t \gg 1$ and, therefore, the normalized inner radius tend to the Newtonian value for large times given as the solution of the algebraic equation

$q\left(\frac{1}{x_{1}}\right)^{3 \gamma}-\frac{2 W e_{1}}{x_{1}}-\frac{2 W e_{2}}{\left(x_{1}^{3}+d^{3}-1\right)^{1 / 3}}-1=0$.

The more relevant parameter to characterize the elasticity of the fluid layer is the Deborah number, defined by the quotient between the relaxation time of the fluid and the characteristic time of the process. His explicit influence is illustrated in Figure $3 \mathrm{ab}$, where normalized radius versus normalized time is plotted for $D e=0.1,1,5$, a shell Reynolds number $\operatorname{Re}_{s}=0.04(\eta=0.5 \mathrm{Pas})$ and a layer thickness $R_{2}-R_{1}=2 \mathrm{~nm}$ (Figure $3 \mathrm{a}$ ) and for $D e=0.1,0.5,1$, a shell Reynolds numbers $R e_{s}=0.008$ $(\eta=1.75$ Pas $)$ and a layer thickness $R_{2}-R_{1}=10 \mathrm{~nm}$ in Figure 3b. As the Deborah number increases, the viscoelastic character of the fluid is enhanced and, correspondingly, oscillatory collapse is more clearly observed, as it is shown in Figure 3a. It may be also clearly recognized in Figure 3b, that at the early stages of the collapse process, the collapse rate is increased as $D e$ increases. This is just the opposite behavior to one would expect, tacking into account that the fluid flow induced by the bubble collapse is an unsteady uniaxial extension flow for which, the extensional resistance should be larger in a viscoelastic fluid layer than that corresponding to a Newtonian fluid layer. In fact, it is easy to show that, in steady uniaxial flow, the constitutive equation (11) predicts an increase in the elongational viscosity as $D e$ increases. This situation reproduces the one found for bubble collapse in viscoelastic fluids $[36,37,38,39,40,41]$, where it is predicted that fluid elasticity accelerates the early stages of the collapse and retards the final stages of the process. Indeed, as described by Zana and Leal [39], the bubble is set into motion from an equilibrium rest state and, initially, there is a period of stress growth where the instantaneous resistance to motion is lesser than the one corresponding to a steady uniaxial flow at the same elongational rate. Consequently, at the early stages of the process, the unsteady collapse rate overshoots the corresponding steady state value. By contrast, in the last periods of the collapse, a steady flow is approached and the influence of larger values of the elongational viscosity in viscoelastic fluid layers becomes evident. It is wort mentioning, the similarity between bubble collapse of a free bubble in a viscoelastic medium and the collapse of a bubble coated by a viscoelastic layer in a Newtonian fluid.

Discrepancies with respect to the Newtonian behavior may be also quantified by means of the rheological parameter $\varepsilon=\lambda_{2} / \lambda_{1}$, as it is decreased from $\varepsilon=1$ (Newtonian fluid). Results for fixed $D e=0.5, \eta=0.5 \mathrm{~Pa}$ s, and the values: $\varepsilon=1 / 9,0.3,0.6,1$ are shown in Figure 4. Here again, oscillatory collapse is induced as $\varepsilon$ decreases. It may be also observed that at the early stages of the process, the collapse rate increases as $\varepsilon$ decreases, in contrast to the behavior expected in view of the increase of the elongational

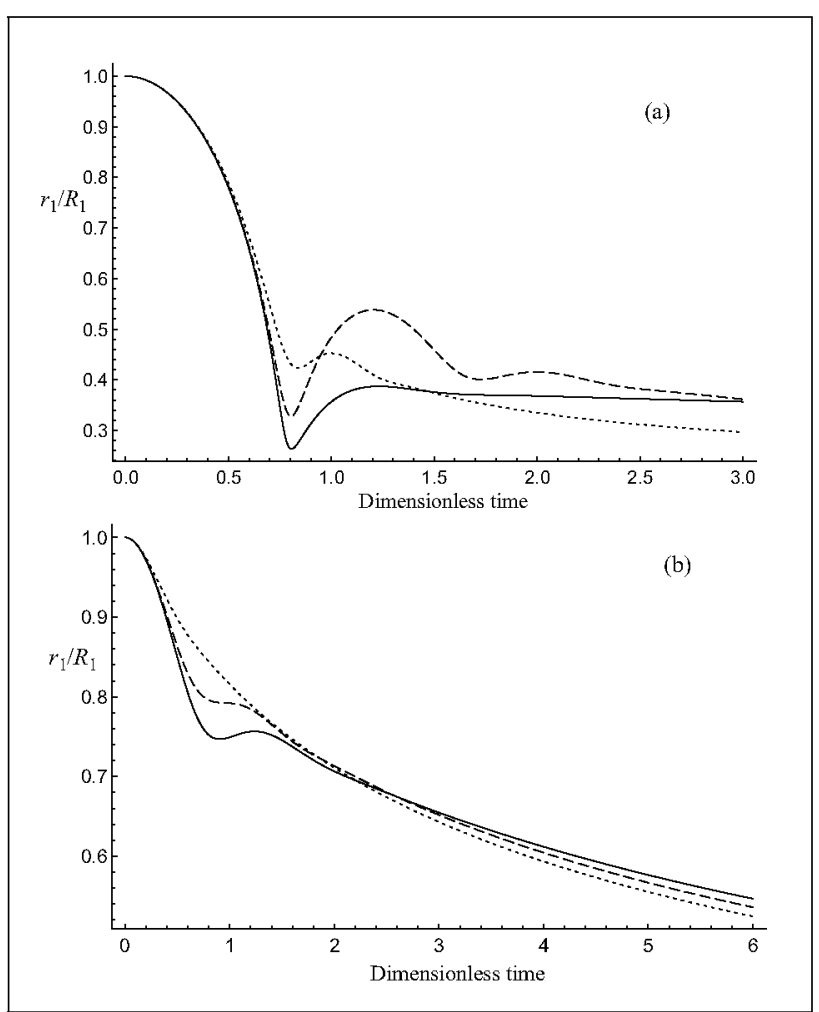

Figure 3. Bubble collapse: influence of the Deborah number, (a) $R e_{s}=0.04, R_{2}-R_{1}=2 \mathrm{~nm}$. Solid line: $D e=5$, dashed line: $D e=1$, dotted line: $D e=0.1$. (b) $R e_{s}=0.008, R_{2}-R_{1}=$ $10 \mathrm{~nm}$. Solid line: $D e=1$, dashed line: $D e=0.5$, dotted line: De $=0.1$.

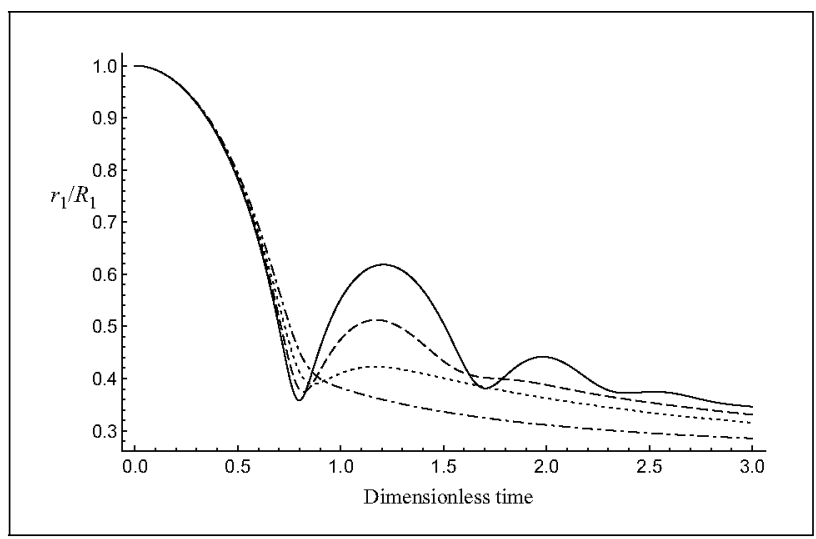

Figure 4. Bubble collapse: influence of the parameter $\varepsilon=\lambda_{2} / \lambda_{1}$. Solid line: $\varepsilon=1 / 9$, dashed line: $\varepsilon=0.3$, dotted line: $\varepsilon=0.6$, dashed line: $\varepsilon=1$ (Newtonian fluid).

viscosity as the parameter $\varepsilon$ is reduced. As noted previously, this apparent anomalous behavior is a consequence of the overshoot of the unsteady collapse rate on the corresponding steady value.

To finish this section, the role played by the rheological parameter $a$ will be analyzed. The constitutive equation (11) predicts an enhancement of the elongational viscosity as the parameter $a$ increases. Accordingly, the collapse radius should be reduced and the collapse rate increased as $a$ growths. This is just the behavior that has been found 


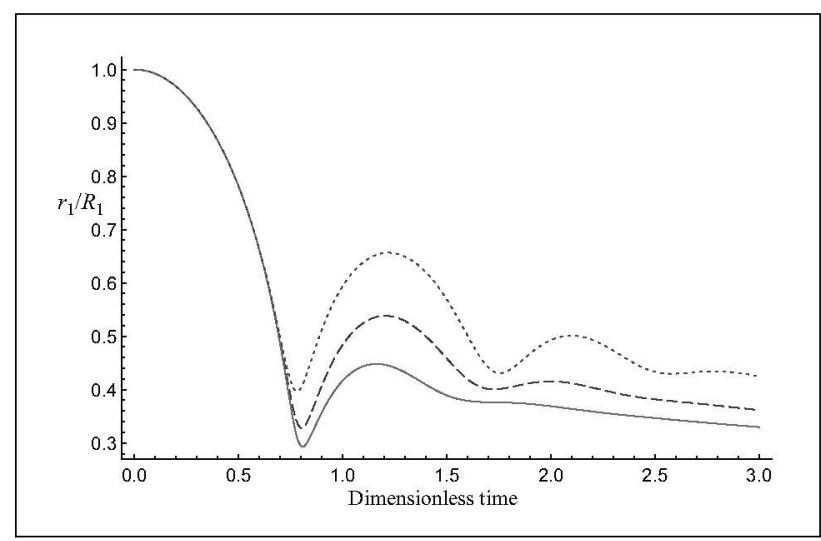

Figure 5. Bubble collapse: influence of the parameter $a$. Solid line $a=-1$ (Oldroyd-A), dashed line $a=1 / 2$, dotted line $a=1$ (Oldroyd-B).

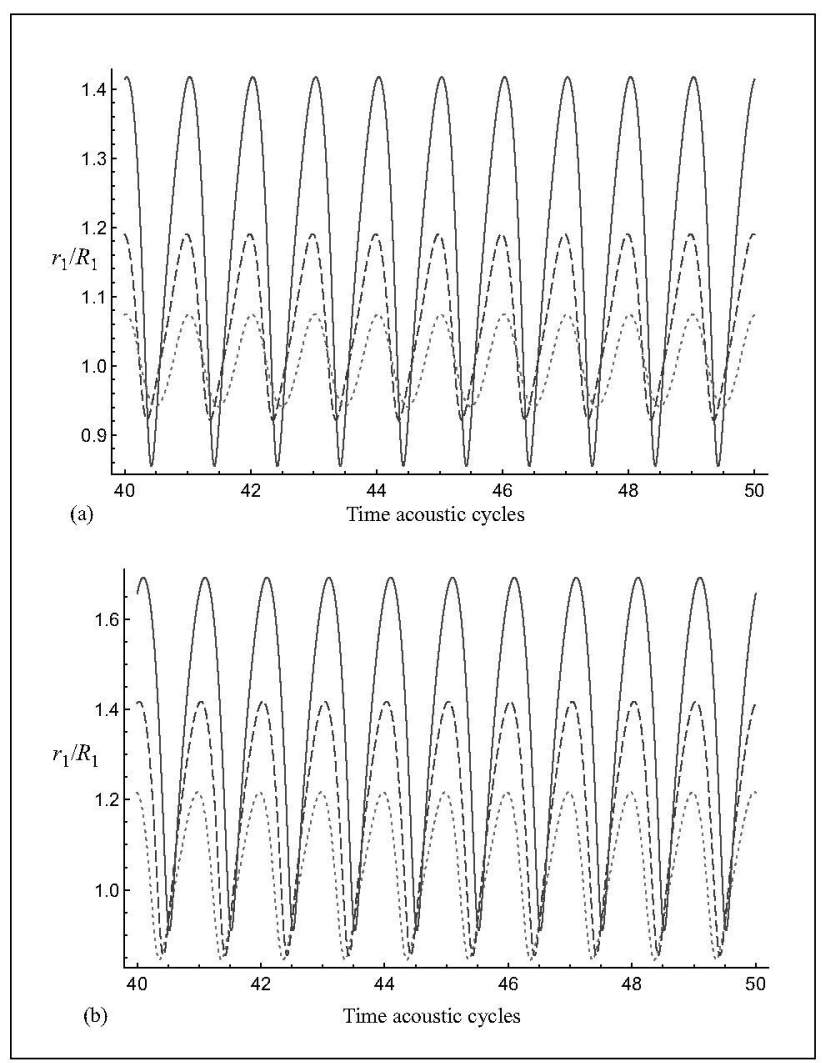

Figure 6. Normalized inner bubble radius versus time in acoustic cycles. (a) solid line $D e=1$, dashed line $D e=0.5$, dotted line: Newtonian. (b) dotted line $a=-1$ (Oldroyd-A), dashed line $a=1 / 2$, solid line $a=1$ (Oldroyd-B).

as it is illustrated in Figure 5, where collapse curves are shown for $a=1$ (Oldroyd B), $a=1 / 2, a=-1$ (Oldroyd A) and $D e=1, \eta=0.5 \mathrm{~Pa} \mathrm{~s}$, respectively. This double dependence of the bubble dynamics on both, the parameter $a$ and the Deborah number will be revisited and discussed in the next section.

\subsection{Dynamic behavior under periodic forcing}

In this section it will be considered that the bubble is subject to the action of a monochromatic wave $p_{\infty}(t)=$
$p_{0}+p_{A} \sin (\omega t)$, where $p_{A}$ is the acoustic pressure amplitude, $\omega=2 \pi f$ the angular frequency and $f$ the acoustic frequency. Numerical results will be obtained for microbubbles immersed in water (data for the host fluid as in section 3.1) with an initial inner radius $R_{1}=2 \mu \mathrm{m}$, a layer thickness $R_{2}-R_{1}=2 \mathrm{~nm}$ (except Figure 6 ) and driven by an acoustic wave with $f=2 \mathrm{MHz}$, a typical value in diagnostic applications. The rest of parameter values will be specified for each case. Bubble radius versus time curves are shown in Figure 6 for bubbles with a layer thickness $R_{2}-R_{1}=20 \mathrm{~nm}$, shell viscosity $\eta=1.5 \mathrm{Pas}$, and pressure amplitude $p_{A}=150 \mathrm{kPa}$. Curves for Deborah numbers $D e=0,0.5,1$, are shown in Figure 6a, respectively. It may be observed that, as it was described in section 3.1 for the collapse problem, fluid elasticity of the shell produces a dramatic increase of the radial amplitude, specially for $D e=1$. The influence of the rheological parameter $a$ is shown in Figure $6 \mathrm{~b}$ where inner radius versus time is plotted for $D e=1$ and $a=-1$ (Oldroyd-A), $a=1 / 2, a=1$ (Oldroyd-B). Note that as $a$ goes from $a=-1$ to $a=1$, the amplitude of the maximal radial expansion is considerably increased. Note also that, conversely, the minimum radius for $a=-1$ is smaller (larger in absolute value) than the corresponding to $a=1$ with an intermediate value for $a=1 / 2$. This finding that has been previously discussed for the collapse problem, deserves special attention. According to the predictions of the constitutive equation (11) for steady extensional flows, the elongational viscosity increases with the parameter $a$ in uniaxial flows and decreases with $a$ in biaxial flow. Consequently, it should be expected that the elongational viscosity increases as the parameter a goes from $a=-1$ to $a=1$ in the uniaxial compression stages, and decreases in the biaxial expansion phases. Thus, minimum radii should be larger for $a=1$ than those for $a=1 / 2$, and $a=-1$, whereas the inverse behavior should be observed for the maximum radial amplitudes. This is just the behavior predicted in the present analysis as it is illustrated in Figure 6b.

\subsection{Regular and chaotic behavior}

The dynamic behavior of an encapsulated microbubble with a shell viscosity $\eta=1 \mathrm{~Pa}$ s and $a=1 / 2$, has been analyzed for an acoustic forcing $p_{A}=350 \mathrm{kPa}$, by increasing the Deborah number from $D e=0$ (Newtonian case) to $D e=0.5$. In Figure 7 normalized inner radius $r_{1} / R_{1}$ versus time (left column) as well as Poincaré section plots (right column) are shown. In these ones, the normalized bubble wall velocity $\dot{r}_{1 p} t_{c} / R_{1}$ is plotted versus the normalized radius $r_{1 p} / R_{1}$, where $r_{1 p}$ is the inner bubble radius at the end of each acoustic cycle. The results show doubling period and transition to chaos as the Deborah number is increased, a behavior that is more clearly illustrated in the bifurcation diagram with the Deborah number as control parameter shown in Figure 8 (data as in Figure 7). A similar behavior was predicted and described for a free bubble immerged in a viscoelastic fluid $[42,43]$. Recently, dynamic system theory has been applied in a comprehensive analysis of the nonlinear response of encapsulated microbub- 


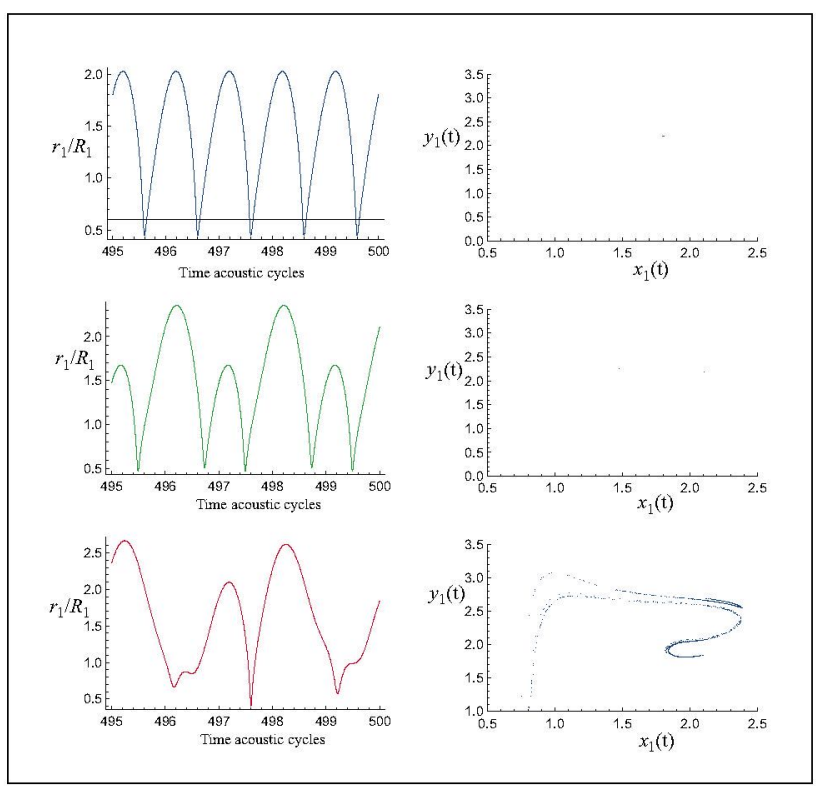

Figure 7. Left column: Normalized inner bubble radius $r_{1} / R_{1}$ versus time in acoustic cycles $\eta=1 \mathrm{Pas}, f=2 \mathrm{MHz} . p_{A}=$ $350 \mathrm{kPa}$. Right column: Poincaré section plot. From top to bottom: $D e=0$ (Newtonian case). $D e=0.05$ and $D e=0.5$.

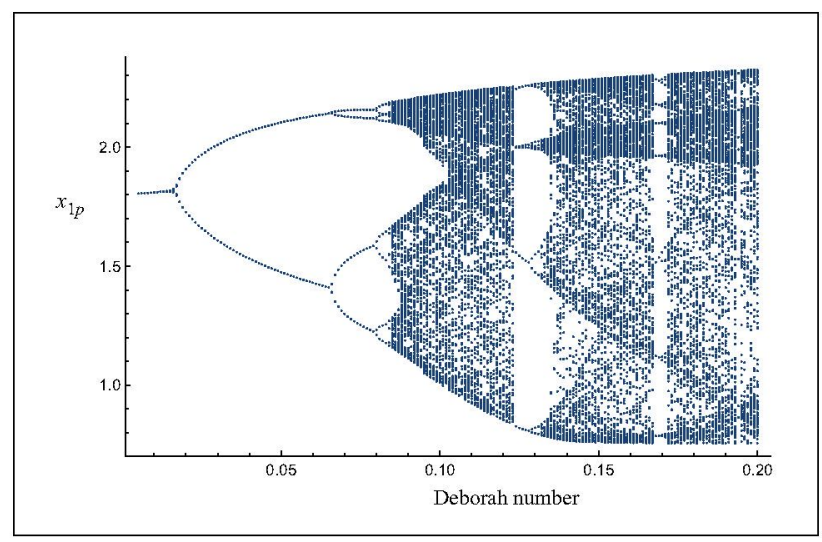

Figure 8. Bifurcation diagrams with the Deborah number as control parameter.

bles [44]. It is not the purpose of this work to perform an exhaustive study of the dynamic problem here considered and outlined in section 2, nevertheless, the results provided in this section, may illustrate the important role played by the fluid elasticity (Deborah number) in the nonlinear responses of this complex system. Bifurcation diagrams with the pressure amplitude as control parameter are shown in Figure 9 a for $D e=1$ and for $D e=0$ (Newtonian case) in Figure 9b. In order to accomplish a direct comparison, the bifurcation diagram of a free-bubble of the same size and driven at the same frequency is plotted in Figure 9c (data as in Figure 7 with $\eta=0.75 \mathrm{Pas}$ ). Note that for a viscoelastic coated bubble the transition to chaos occurs at pressure amplitudes above those that produce chaotic oscillations for a free-bubble. In other words, a microbubble encapsulated by a viscoelastic fluid shell experiences regu-

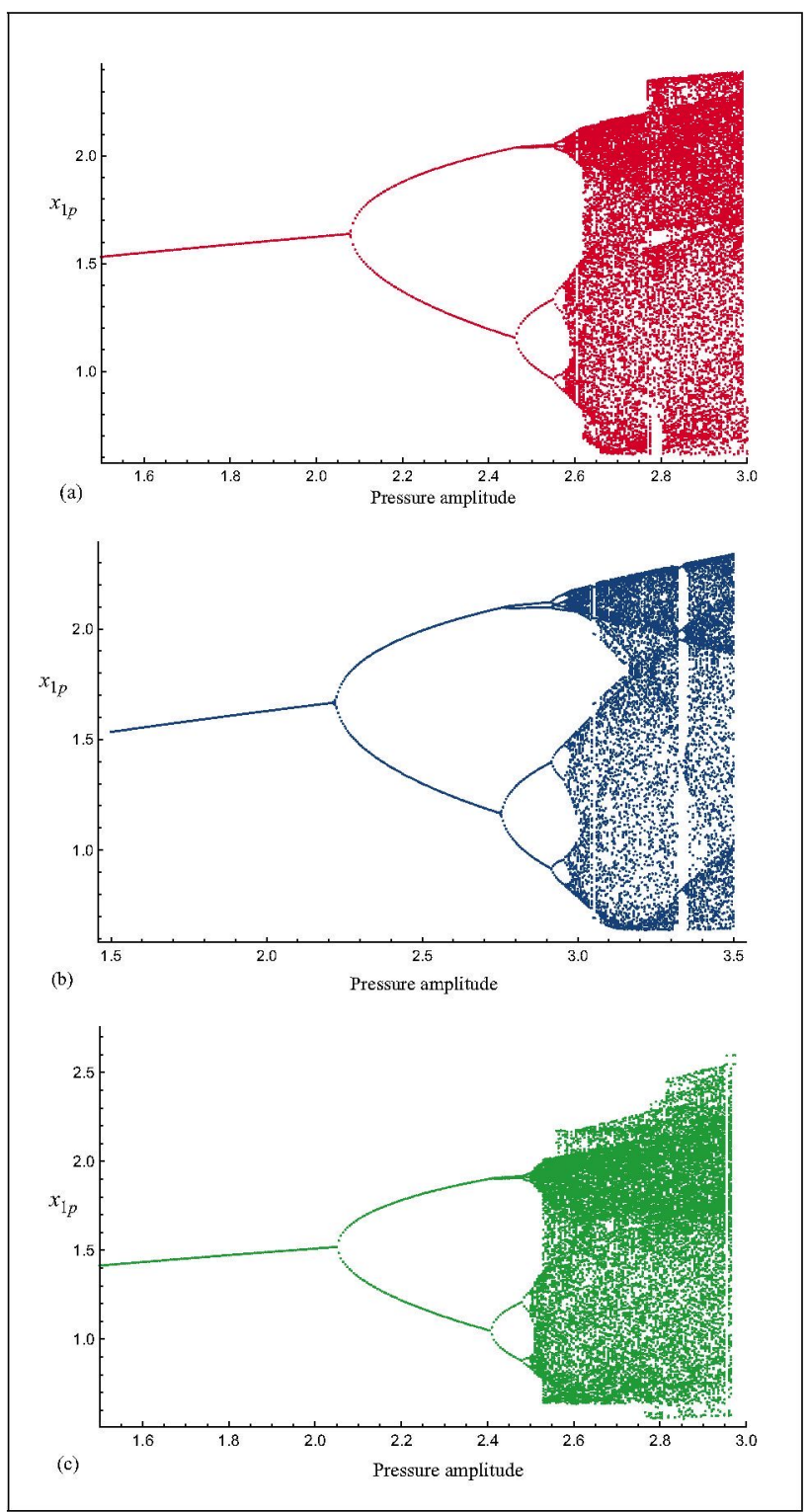

Figure 9. Bifurcation diagrams with acoustic pressure as control parameter. (a) viscoelastic fluid shell with $D e=1$ (b) Newtonian fluid shell, (c) free-bubble.

lar periodic radial oscillations at pressures that drive a free bubble into chaotic oscillations.

\subsection{Maximum relative expansion}

It has been shown in previous sections that under acoustic driving, bubbles encapsulated by a viscoelastic fluid shell undergo radial oscillations of considerably large amplitude, a result that may be of interest in diagnosis imaging. Accordingly, the relative importance of the governing parameters on the maximum amplitude expansion will be analyzed in detail. Thus, in this section, the quantitative influence of the driving pressure amplitude, the Deborah number, the shell Reynolds number and the shell thickness, will be subsequently estimated. In the following, numerical calculations will be performed by considering $a=1 / 2$, and the fixed parameter values quoted in section 3.2 . 


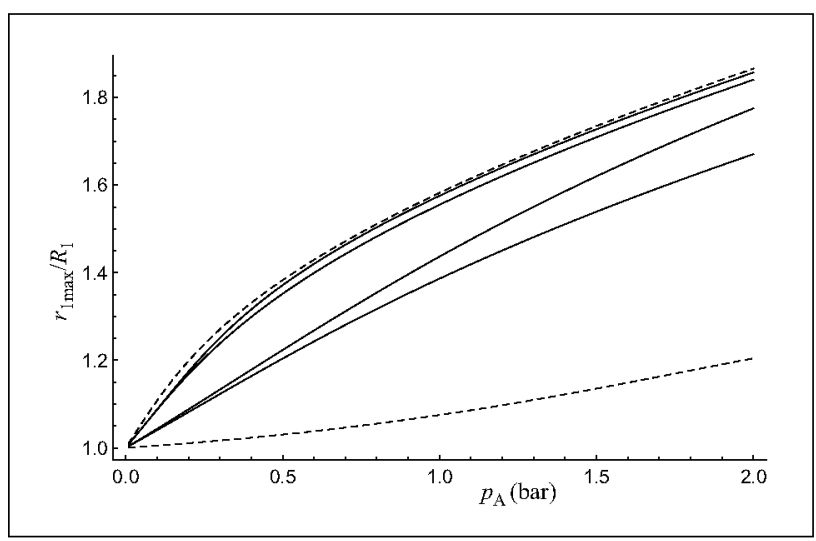

Figure 10. Normalized maximum radial amplitude $r_{1 \max } / R_{1}$ versus acoustic pressure $p_{A}$ : blue line: Newtonian $(D e=0)$, green line: $D e=0.1$, red line: $D e=1$. Blue dashed line: Kelvin-Voigt solid ( $G=166.7 \mathrm{MPa}$ ), red dashed line: free bubble.

The maximal radial amplitude $r_{1 \max } / R_{1}$ as a function of the pressure $p_{A}$ for different values of $D e$ and a shell Reynolds number $R e_{s}=0.017$ ( $\eta=1.2$ Pas) is shown in Figure 10. For the sake of comparison, the corresponding curves for a solid shell (Kelvin-Voigt model) and a free bubble have also been plotted in this figure. The quantitative differences in radial expansion between solid coating and fluid coating are evident. For liquid shells, the influence of $D e$ becomes particularly apparent as the pressure amplitude increases. Note, however, the minute differences that may be distinguished for $D e=1$, and $D e=10$ or larger values (not plotted) with respect to a free bubble. In fact, for any pressure amplitude, $r_{1 \max } / R_{1}$ increases with $D e$, reaches a maximum value for $D e \approx 1$ which is close to the one corresponding to a free bubble and then decreases to an asymptotic value for $D e \gg 1$, as it is illustrated in Figure 11 where $r_{1 \max } / R_{1} v s \quad D e$ is plotted for $\operatorname{Re}_{s}=0.01(\eta=2 \mathrm{Pas})$ and pressure amplitudes $p_{A}=50,100,200 \mathrm{kPa}$. The asymptotic behavior for large De may be determined from expressions (42)-(43) for the normal stress difference in dimensionless form. Indeed, for De $\gg 1$, the following analytical expression has been obtained:

$$
S=S_{1}+S_{2}=\frac{2(1-\varepsilon)}{D e R e_{S}}\left(\frac{1}{x_{1}^{2}}-\frac{d^{2}}{x_{2}^{2}}\right)
$$

This expression shows that the asymptotic behavior of the normal stress difference for large $D e$ is controlled by $\left(D e R e_{s}\right)^{-1}$, so that, for moderate or large $R e_{s}$ values, the viscoelastic coating approaches the behavior of a Newtonian fluid shell (see expression 40).

The influence of the shell viscosity is illustrated in Figure 12 , where the maximal radial amplitude is plotted versus the Reynolds number of the layer $\boldsymbol{R e}_{S}$ for $D e=$ $0,0.1,1$ and a pressure amplitude $p_{A}=100 \mathrm{kPa}$. For small Deborah numbers, a significant dependence of the maximal radial amplitude on $R e_{s}$ is detected. However, for $D e=1$ or larger the influence of $R e_{s}$ is relatively small. In other words, for Deborah numbers of the order of unity

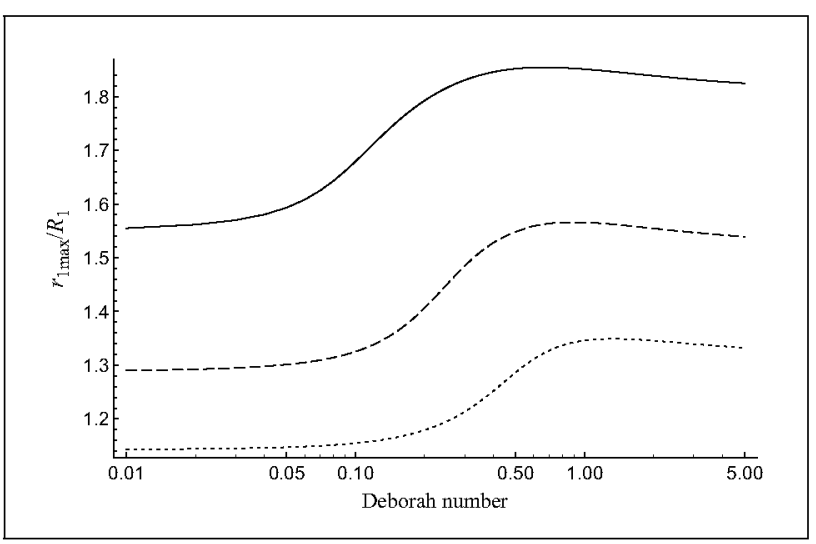

Figure 11. Normalized maximum radial amplitude $r_{1 \max } / R_{1}$ versus Deborah number: Dotted line: $p_{A}=50 \mathrm{kPa}$, dashed line: $p_{A}=100 \mathrm{kPa}$, solid line: $p_{A}=200 \mathrm{kPa}$.

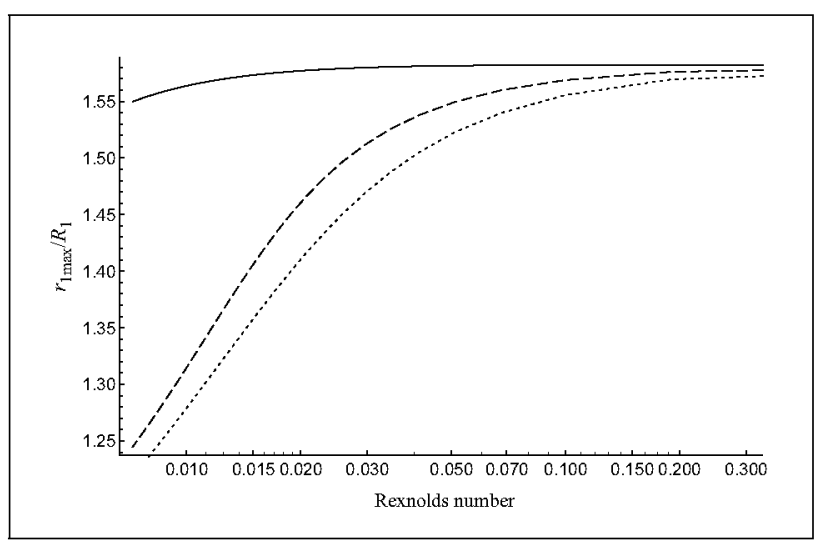

Figure 12. Normalized maximum radial amplitude $r_{1 \max } / R_{1}$ versus the Reynolds number of the shell $R e_{s}$. Dotted line Newtonian $(D e=0)$, dashed line: $D e=0.1$, solid line: $D e=1$ and $p_{A}=100 \mathrm{kPa}$.

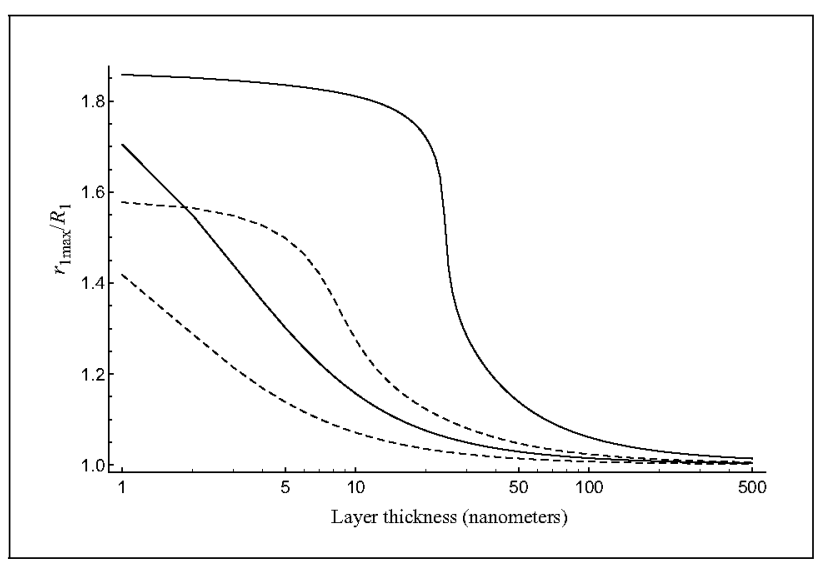

Figure 13. Normalized maximum radial amplitude $r_{1 \max } / R_{1}$ versus the thickness layer $R_{2}-R_{1}$. Blue lines (lower solid and dashed curve) Newtonian ( $D e=0$ ), red (upper solid and dashed curve) lines: $D e=1$. Solid lines correspond to $p_{A}=200 \mathrm{kPa}$ and dashed lines to $p_{A}=100 \mathrm{kPa}$.

or larger, damping effects are irrelevant and the bubble dynamics is governed by elastic effects. 
Above results have been obtained by considering a small layer thickness $R_{2}-R_{1}=2 n m$, however, this parameter may have, in the viscoelastic case, a considerable quantitative influence as it is shown in Figure 13, where $r_{1 \max } / R_{1}$ is plotted versus the layer thickness for $R e_{s}=0.01(\eta=2$ Pa.s $)$ pressure amplitudes $p_{A}=100$, $200 \mathrm{kPa}$ and $D e=0,1$. Note that for small values of $D e$ or in the Newtonian case ( $D e=0), r_{1 \max } / R_{1}$ decreases smoothly as the layer thickness increases. However, for $D e=1$, and mainly at high pressure amplitudes, the maximal radial expansion remains nearly constant for small thickness but decreases sharply above a given value of this one. A numerical example may illustrate this output: for $D e=1$ and $p_{A}=200 \mathrm{kPa}$ the maximum radial expansion $r_{1 \text { max }} / R_{1}=1.8$ for $R_{2}-R_{1}$ below $25 \mathrm{~nm}$, and is abruptly reduced to $r_{1 \max } / R_{1}=1.2$ for $R_{2}-R_{1}$ above $30 \mathrm{~nm}$.

\section{Conclusions}

The dynamics of a gas bubble encapsulated by a viscoelastic shell and subject to the action of a external pressure field has been investigated. Bubble collapse under a sudden change of the external pressure as well as radial volume oscillations under an ultrasonic monochromatic field has been considered. The main results obtained may summarized as follows:

- Oscillatory collapse is predicted for a viscoelastic shell in conditions where the collapse of a Newtonian shell is monotonous. The elasticity of the fluid shell accelerates the early stages of the collapse and retards the final stages of the process. A behavior similar to the one observed for the bubble collapse of free-bubbles immersed in viscoelastic fluids.

- Shell elasticity enhances the amplitude of radial oscillations which increases dramatically as the Deborah number increases and become chaotic even for moderate values of the external pressure amplitude. Nevertheless, critical values for the onset of bifurcation and chaos for a viscoelastic shell, are smaller than those corresponding to a free bubble.

- The asymmetry of radial oscillations with respect to the initial equilibrium radius evidences the influence of the elongational viscosity. Thus, as the rheological parameter $a$ goes from $a=-1$ (Oldroyd-A) to $a=1 / 2$, and $a=1$ (Oldroyd-B), maximal radii are clearly increased and minimal radii reduced. This result may be explained taking into account that, according to the predictions of the Oldroyd model in extensional flows, as the parameter $a$ increases, the elongational viscosity increases in the uniaxial flow of the collapsing phase whereas decreases in the biaxial flow corresponding to the expansion phase.

- For a value of the driving frequency $f=2 \mathrm{MHz}$, a typical value in diagnosis applications, the maximal amplitude of radial oscillations is observed for Deborah numbers around the unity. In the limit of very large Deborah numbers, an analytical expression for the normal stress difference has been obtained. This expression shows that the asymptotic behavior of the normal stress difference for large $D e$ is controlled by $\left(D e R_{S}\right)^{-1}$, so that, for moderate or large $\boldsymbol{R}_{s}$ values, the viscoelastic coating approaches the behavior of a Newtonian fluid shell. For small Deborah numbers, elastic effects are reduced as the Reynolds number of the layer decreases.

- The influence of the shell thickness has also been analyzed. It has been found that for small Deborah numbers, the maximum radial amplitude decreases smoothly for increasing coating thickness. However, for Deborah numbers of the order of unity, the maximal radial expansion remains nearly constant for small layer thickness but decreases abruptly above a critical value of this one. This critical value increases as the amplitude of the driving pressure is increased.

According to the above theoretical predictions, it may be concluded that a microbubble coated by a viscoelastic fluid shell is an oscillating system that, under acoustic driving, may experience volume oscillations of large amplitude, being, however, more stable than a free bubble. Thus, it could be expected that such a system may have a suitable behavior as an echogenic agent. 